\title{
Estudio transversal de consumo de tabaco en trabajadores de un hospital de tercer nivel
}

\author{
Luis Villalobos-Gallegos', Marcela Tiburcio-Sainz², Ricardo Sánchez-Domínguez', Rodrigo Marín-Navarrete' \\ ' Unidad de Ensayos Clínicos, Subdirección de Investigaciones Clínicas, Instituto Nacional de Psiquiatría Ramón de la Fuente Muñiz, México \\ ${ }^{2}$ Dirección de Investigaciones Epidemiológicas y Psicosociales, Instituto Nacional de Psiquiatría Ramón de la Fuente Muñiz, México
}

\section{RESUMEN}

Introducción: el consumo de tabaco es la primera causa prevenible de muerte y morbilidad en el mundo; estudios previos en hospitales muestran que la población médica sirve como modelo positivo en la promoción de la abstinencia del tabaco, sin embargo, cuando el personal de salud lo usa, las probabilidades de abstinencia disminuyen. Objetivos: 1) conocer la prevalencia de tabaquismo, 2) comparar las distintas áreas de trabajo en función de variables relacionadas con el consumo de tabaco, 3) identificar los factores de riesgo. Método: a 1,004 trabajadores de un establecimiento hospitalario se les aplicó un cuestionario de autorreporte que contenía preguntas para evaluar el tabaquismo, así como el Fagerström Test of Nicotine Dependence (FTND) y la Cigarette Dependence Scale (CSD-12 y CSD-5) para evaluar dependencia de nicotina. Resultados: $23.4 \%$ de los trabajadores consumió tabaco en el último año, $60.4 \%$ de los fumadores son del sexo masculino, $91.2 \%$ presentó dependencia baja. Entre los factores de riesgo se encontraron los siguientes: grupo de edad de 20 a 31 años (OR=2.62, IC del 95\% [1.773.88], $p<.001$ ), ser hombre (OR=2.91, IC del 95\% [1.824.68], $p<.001)$, edad de inicio del consumo (OR=1.09, IC del $95 \%$ [.71-1.68], $p<.001$ ), estado civil (OR=4.51, IC del $95 \%[1.18-17.18], p=.027)$, y tener una escolaridad de secundaria (OR=3.51, IC del 95\% [1.66-7.40], $p=.001) \mathrm{O}$ bachillerato (OR=3.11, IC del 95\% [1.61-6.02], $p=.001)$. Discusión y conclusiones: el presente estudio aporta información actual sobre el consumo de tabaco en trabajadores de planta de un hospital de tercer nivel; se encontró una baja prevalencia de médicos que consumen tabaco (16.3\%), en comparación con el personal de otra áreas de labor. Las implicaciones relacionadas con los hallazgos se discuten en el estudio.

Palabras clave: tabaquismo; trabajadores de hospital; test de Fagerström de dependencia de la nicotina; escala de dependencia de cigarrillos.

\begin{abstract}
Introduction: tobacco use is the leading cause of preventable deaths and morbidity around the globe. Previous studies in medical hospitals population are seen as positive role in promoting on tobacco abstinence, however, when tobacco use in health personnel, the odds decrease withdrawal. Objectives: 1 ) determine the prevalence of smoking, 2) compare different workspaces on variables allied to the tobacco use, 3) identify risk factors. Method: a self-report questionnaire containing questions to assess tobacco use and the Fagerström Test of Nicotine Dependence and the Cigarette Dependence Scale to assess nicotine dependence was applied to 1004 workers in the hospital. Results: $23.4 \%$ of the sample reported tobacco use in the last year, $60.4 \%$ of the smokers are males, $91.2 \%$ with a low dependence. Among the risk factors found that the age group 20 to 31 years ( $O R=2.62$, $95 \% \mathrm{Cl}[1.77-3.88], p<.001)$, male $(\mathrm{OR}=2.91,95 \%$ $\mathrm{Cl}[1.82-4.68], p<.001)$, age of first use $(\mathrm{OR}=1.09,95 \% \mathrm{Cl}$ $[.71-1.68], p<.001)$, civil status (OR=4.51, 95\% Cl [1.18$17.18], p=.027)$, secondary school (OR=3.51, 95\% Cl [1.667.40], $p=.001)$, high school (OR=3.11, 95\% Cl [1.616.02], $p=.001$ ) increase the probability to be regular smoker. Discussion: the present study provides current information of the tobacco use in a third level hospital workers, finding a low prevalence of physicians who smoking behavior (16.3\%) compared to other areas of work, the related implications with the findings discussed in the study.
\end{abstract}

Keywords: smoking; hospital workers; Fagerström test for nicotine dependence; cigarette dependence scale.

\footnotetext{
Autor de correspondencia:

Luis Villalobos-Gallegos. Instituto Nacional de Psiquiatría Ramón de la Fuente Muñiz, Unidad de Ensayos Clínicos en Adicciones y Salud Mental. Calzada México-Xochimilco núm. 101, col. San Lorenzo Huipulco, del. Tlalpan, C.P. 14370, Ciudad de México. Tel.: +52 (55) 4160-5482. Correo electrónico: Ivillalobos@imp.edu.mx

Recibido: 21 de septiembre de 2016.

Aceptado: 6 de octubre de 2016.

DOI: 10.28931/riiad.2016.2.04
} 


\section{INTRODUCCIÓN}

El tabaquismo es la principal causa prevenible de muerte y morbilidad en el mundo (Organización Mundial de la Salud [OMS], 2015) y el cuarto factor de riesgo de pérdida de años de vida (Murray \& Lopez, 1997). Genera un riesgo importante en el desarrollo de diversas enfermedades con consecuencias fatales como cáncer, enfermedad pulmonar crónica obstructiva y enfermedades isquémicas, entre otras (OMS, 2015).

Los daños a la salud producidos por esta epidemia provocan un importante gasto en atención médica, pérdidas en el área laboral y mortalidad en diversas etapas de desarrollo; de acuerdo con la OMS (2003), los daños económicos ocasionados por el tabaco ascienden a 500 mil millones de dólares anuales aproximadamente. En México, al año se gastan 75 mil 200 millones de pesos en la atención de problemas relacionados con el tabaco, esto sin tomar en cuenta las mermas económicas derivadas del ausentismo, del tiempo laboral perdido por salir a fumar y el tiempo laboral perdido por asistencia a servicios de salud (Guerrero-López et al., 2012; Waters, Sáenz-de Miera, Ross, \& Reynales, 2010).

Estudios recientes han encontrado que el personal médico de los hospitales sirve como modelo positivo; sin embargo, si el profesional de la salud o de otras áreas del hospital presenta consumo de tabaco, las acciones que realiza para promover la abstinencia en pacientes fumadores tienen un impacto significativamente menor (Maeckelberghe, 2013; Ravara, Castelo-Branco, Aguiar, \& Calheiros, 2014), sobre todo en lo referente al establecimiento de planes para dejar de fumar y a la prescripción de medicamentos (Pipe, Sorensen, \& Reid, 2009), ya que el uso de tabaco por parte del personal de salud genera un escepticismo que conduce a una poca adherencia y al abandono del tratamiento (Chapman, 1995; Duaso, McDermott, Mujika, Purssell, \& While, 2014).

Ante esta problemática, se han realizado diversos estudios para conocer la prevalencia de consumo entre trabajadores de hospitales (Abdulateef, Ali, Abdulateef, \& Mohesh, 2016; Al-Hosani et al., 2014); se encontraron diferencias en el uso de tabaco por parte del personal médico y del personal de salud no médico (Martínez et al., 2016; Tong, Strouse, Hall, Kovac, \& Schroeder, 2010), así como entre el personal de salud (incluyendo médicos) y el de otras áreas del hospital (Lewis, Shin, \& Davies, 2011).

En México son pocos los estudios que exploran el uso de tabaco por parte del personal del hospital (Arenas, Hernández, Jasso, Martínez, \& Menjivar, 2004; Salmerón-Castro, Arillo-Santillán, Campuzano-Rincón, López-Antuñano, \& Lazcano-Ponce, 2002); algunas investigaciones han explorado factores de riesgo para que el personal padezca enfermedades cardiovasculares (Orozco-González, Cortes-Sanabria, Viera-Franco, Ramirez-Márquez, \& Cueto-Manzano, 2016), mientras que otras se han ocupado del perfil de salud de los trabajadores (Velasco-Contreras, 2013); no obstante, no se ha estudiado a detalle el consumo de tabaco y los factores de riesgo relacionados con el mismo.

Estudios previos han reportado que los factores que predicen un aumento en el consumo de tabaco son el género (Stone, Becker, Huber, \& Catalano, 2012), la edad de inicio de consumo (Villatoro-Velázquez et al., 2016), el estado civil (Cho, Khang, Jun, \& Kawachi, 2008) y el nivel de estudios (Carter et al., 2015), entre otros. Ya que este tipo de estudios no se realiza con regularidad y no se cuenta con información reciente, es importante tanto el monitoreo del uso de tabaco por parte del personal de salud como de los factores de riesgo asociados, a fin de implementar estrategias de prevención.

Por tal motivo, este trabajo tiene tres objetivos: 1) conocer la prevalencia de tabaquismo en los trabajadores de un hospital de tercer nivel; 2) comparar las distintas áreas de trabajo en el hospital en función de variables relacionadas con el consumo de tabaco; y 3 ) identificar los factores de riesgo relacionados con el uso de la sustancia.

\section{MÉTODO}

\section{Diseño del estudio}

Se trata de una investigación primaria, epidemiológica, observacional, transversal (Röhrig, du Prel, Wachtlin, \& Blettner, 2009).

\section{Participantes}

Se realizó un muestreo intencional. La muestra estuvo conformada por personal de las diferentes áreas del hospital: médicos, atención a la salud (no médicos) y administrativos/servicios generales. Los criterios de inclusión fueron: ser trabajador del hospital (sin importar el sexo) y firmar un consentimiento de participación. La muestra analizable fue de 1,004 hombres y mujeres trabajadores de planta.

\section{Medidas}

Se utilizó un cuestionario de autorreporte con preguntas que indagaron las siguientes variables: demográficas (edad, sexo, estado civil, nivel académico y años de estudios), prevalencia de consumo de tabaco ("¿Fuma usted actualmente?", "¿a qué edad inicio el consumo regular de cigarrillos?"), patrón de consumo ocasional (“¿Qué tan seguido fuma?"), edad de inicio ("¿A qué 
edad probó por primera vez un cigarrillo?"), edad de cesación ("¿A qué edad dejó de fumar?") y nivel de dependencia de la nicotina.

Para medir esta última variable se aplicaron tres cuestionarios. El primero fue el Fagerström Test of Nicotine Dependence [FTND] (Heatherton, Kozlowski, Frecker, \& Fagerstrom, 1991), conformado por seis reactivos, cuatro ítems con calificación de 0 a 1 y dos ítems de elección múltiple con calificación de 0 a 3; los elementos se sumaron para dar una puntuación total de 0-10, con un $a=.61$. Cabe destacar que cuanto mayor sea la puntuación total de Fagerström, más intensa es la dependencia física del paciente a la nicotina.

El segundo cuestionario fue el Cigarette Dependence Scale (CDS-12 items) y el tercero, el CDS-5 ítems (versión corta) (Etter, Le Houezec, \& Perneger, 2003), con opciones de respuesta del 1 al 5 . Los resultados se sumaron; cuanto mayor fue el puntaje, mayor fue la dependencia. Ambas escalas presentan una alta confiabilidad test-retest $(r=.83)$ y una alta consistencia interna $(a=.84)$. Ambos instrumentos cubren los principales indicadores del DSM-IV y la CIE-10 de dependencia de la nicotina: compulsión, abstinencia, pérdida de control, abandono de actividades, persistencia a pesar del daño.

\section{Procedimientos}

El levantamiento de los datos se llevó a cabo en colaboración con la Subdirección de Salud Pública y el personal administrativo del hospital. Los cuestionarios se entregaron dos días antes de la aplicación, en el área donde se realiza la firma de nómina; los miembros del departamento de pagos entregaron el formato de consentimiento y el cuestionario a cada una de las personas que asistieron a recoger su pago. Después de leer la información relativa al estudio y firmar el consentimiento, los participantes contestaron el cuestionario; finalizada la aplicación, se recogieron los formularios para su resguardo y la asignación de un número de folio. Los datos fueron levantados entre enero y marzo de 2010.

Los materiales utilizados en el estudio fueron revisados y aprobados por la Subdirección de Salud Pública y el Comité de Ética del hospital. Todos los participantes del estudio firmaron un consentimiento informado para garantizar su participación voluntaria.

\section{Análisis estadístico}

Se realizaron análisis descriptivos para variables demográficas (edad, sexo, grado de estudios, estado civil). Pruebas $t$ de Student para variables numéricas y $X^{2}$ para variables categóricas que comparan entre hombres y mujeres. Con la finalidad de lograr el segundo objetivo, se aplicó análisis de varianza de un factor (ANOVA) para comparar al grupo de médicos con personal de salud no médico y personal de otros servicios, con variables numéricas y $X^{2}$ para variables categóricas. Para responder al tercer objetivo, estimando la probabilidad de que un grupo presente o no un evento especifico, se utilizó el análisis de regresión logística multinomial debido a que la variable de resultado es politómica (fumador regular, fumador ocasional, exfumador y no fumador). En este tipo de regresión, los resultados se obtienen en función de un grupo de comparación, el de no fumadores; se obtuvo la razón de momios (OR) entre las variables de exposición, las cuales pueden ser factores o covariables. Los análisis se realizaron mediante el paquete estadístico SPSS versión 23 para Windows.

\section{RESULTADOS}

\section{Características de los participantes}

Un total de 1,278 personas laboran en el hospital, de las cuales 238 no aceptaron participar; también se eliminaron aquellas que respondieron menos de $20 \%$ de los reactivos, con lo que quedaron 1,004 participantes en la muestra final (Figura 1).

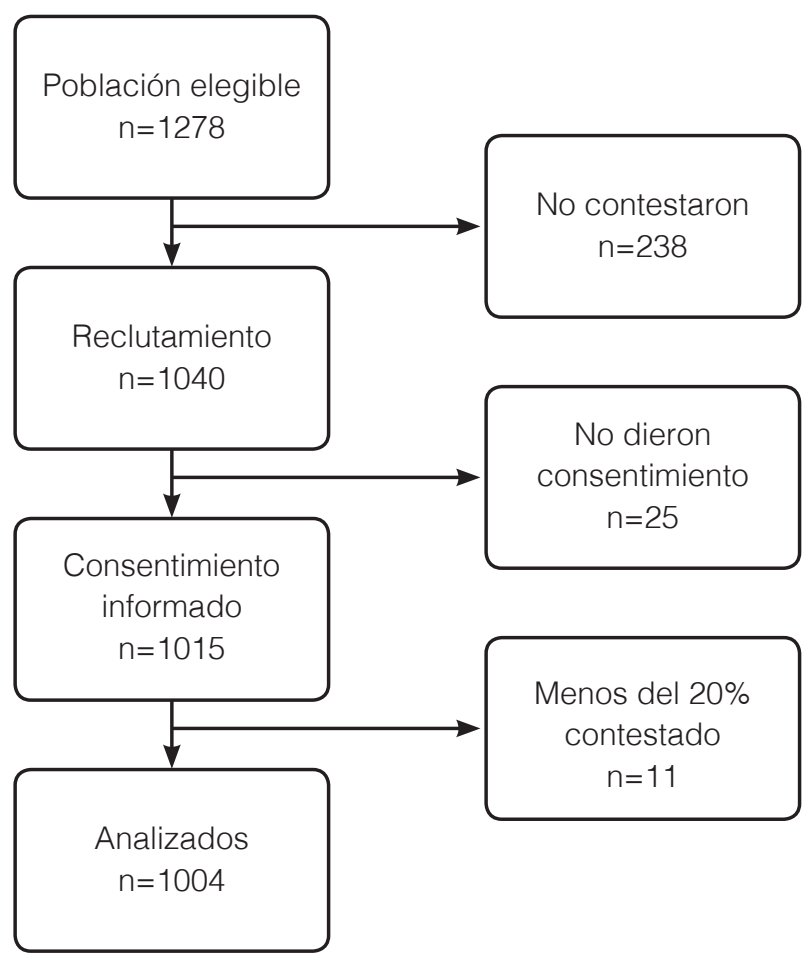

Figura 1. Tasa de respuesta a través del estudio 
$57.3 \%$ de la muestra estuvo conformada por mujeres y $42.6 \%$ por hombres. En mujeres se presentó una media de edad de 32 años ( $D E=7.1$ ) , 7.4 años ( $D E=6$ ) de antigüedad laboral y 13.8 años ( $D E=3.7)$ de estudio; datos similares se encontraron en hombres: edad de 32.2 años ( $D E=7.2$ ), 7.2 años de antigüedad (6.7) y 14 años (4.7) de estudio.

Se encontró que $51.3 \%$ de los fumadores ocasionales y $60.4 \%$ de los regulares son del género mas- culino; $58.0 \%$ de las mujeres no ha fumado y $22.0 \%$ son exfumadoras. $47.3 \%$ de la mujeres y $41.8 \%$ de los hombres tienen escolaridad media superior; $46.4 \%$ y $51.2 \%$ (mujeres y hombres, respectivamente) trabajan en el área de servicios y administración. Se observó que $44.5 \%$ de las mujeres pertenece al área de salud no médica, en comparación con el 31.3\% de los hombres (Tabla 1).

Tabla 1

Variables sociodemográficas de los participantes y comparación por sexo

\begin{tabular}{|c|c|c|c|c|}
\hline & Mujeres & Hombres & Total & \\
\hline & $n=562^{a}$ & $n=418^{a}$ & $n=1004$ & estadística \\
\hline & $M(\mathrm{DE})$ & $M(\mathrm{DE})$ & $M(\mathrm{DE})$ & \\
\hline Edad: & $32.0(7.1)$ & $32.2(7.2)$ & $32.1(7.4)$ & $t_{(943)}=.71$ \\
\hline Antigüedad laboral (años) & $7.4(6.0)$ & $7.2(6.7)$ & $7.3(6.3)$ & $t_{(924)}=.58$ \\
\hline Años de estudio & $13.8(3.7)$ & $14.0(4.7)$ & $13.8(4.3)$ & $t(860)=-.60$ \\
\hline Edad de primer consumo & $17.7(3.7)$ & $16.9(3.3)$ & $17.3(3.6)$ & $t_{(426)}=2.35^{*}$ \\
\hline Edad de consumo regular & $20.3(5.4)$ & $20.4(4.5)$ & $20.3(4.9)$ & $t_{(141)}=-.20$ \\
\hline \multirow[t]{2}{*}{ Edad en que dejó de fumar } & $24.2(6.3)$ & $23.9(7.1)$ & $24.1(6.6)$ & $t_{(146)}=.26$ \\
\hline & $\mathrm{n}(\%)$ & $\mathrm{n}(\%)$ & $\mathrm{n}(\%)$ & \\
\hline \multicolumn{5}{|l|}{ Escolaridad } \\
\hline Primaria & $2(.4)$ & $2(.52)$ & $4(.44)$ & \\
\hline Secundaria & $63(12.3)$ & $80(20.7)$ & $143(15.9)$ & \\
\hline Bachillerato & $243(47.3)$ & $161(41.8)$ & $404(45)$ & $X^{2}{ }_{(4)}=17.52^{*}$ \\
\hline Licenciatura & $120(23.3)$ & $65(16.8)$ & $185(20.5)$ & \\
\hline Posgrado & $85(16.5)$ & $77(20.0)$ & $162(17.9)$ & \\
\hline \multicolumn{5}{|l|}{ Tipo de actividad laboral } \\
\hline Médicos & $51(9.1)$ & $73(17.5)$ & $124(12.7)$ & \\
\hline Salud no médicos & $250(44.5)$ & $131(31.3)$ & $381(38.9)$ & $X^{2}{ }_{(2)}=25.10^{*}$ \\
\hline Servicios y administrativos & $261(46.4)$ & $214(51.2)$ & $475(48.5)$ & \\
\hline \multicolumn{5}{|l|}{ Estado civil } \\
\hline Soltero & $269(50.4)$ & $131(32.8)$ & $400(42.9)$ & \\
\hline Casado/unión libre & $257(48.1)$ & $260(65.2)$ & $517(55.4)$ & $X^{2}(2)=28.69^{*}$ \\
\hline Separado/divorciado/viudo & $8(1.5)$ & $8(2.0)$ & $16(1.7)$ & \\
\hline Consumo en el último año & $107(19.9)$ & $129(33.2)$ & $236(25.4)$ & $X^{2}(1)=21.10^{*}$ \\
\hline \multicolumn{5}{|l|}{ Estatus de fumador } \\
\hline Fumador regular & $34(6.3)$ & $52(13.4)$ & $86(9.3)$ & \\
\hline Fumador ocasional & $73(13.5)$ & $77(19.8)$ & $150(16.2)$ & $X^{2}(3)=29.40^{*}$ \\
\hline Exfumador & $119(22.0)$ & $96(24.6)$ & $215(23.2)$ & \\
\hline No fumador & $313(58.0)$ & $164(42.1)$ & $477(51.5)$ & \\
\hline \multicolumn{5}{|l|}{ Patrón de consumo } \\
\hline Menos de una vez al mes & $18(26.1)$ & $14(19.2)$ & $32(22.4)$ & \\
\hline Una vez al mes & $6(8.7)$ & $3(4.1)$ & $9(6.8)$ & \\
\hline 2 a 3 veces por mes & $13(18.8)$ & $17(23.2)$ & $30(21.0)$ & $X^{2}(4)=2.82$ \\
\hline Una vez a la semana & $13(18.8)$ & $18(24.6)$ & $31(21.7)$ & \\
\hline 2 a 3 días a la semana & $19(27.5)$ & $21(28.7)$ & $40(27.2)$ & \\
\hline
\end{tabular}


Los hombres iniciaron su consumo a edad más temprana (16.9 años), en comparación con las mujeres (17.7 años). También se encontró una mayor prevalencia en el patrón de consumo de tabaco, se observó que $28.7 \%$ fuma de dos a tres días a la semana, 24\% lo hace una vez a la semana y $23.2 \%$ de dos a tres veces por mes.

Se encontraron diferencias estadísticamente significativas entre hombres y mujeres en la edad de primer consumo $\left(t_{(426)}=2.35, p=.019\right)$, el nivel de estudios $\left(X^{2}{ }_{(4)}=\right.$ 17.52, $p=.002)$, el tipo de actividad laboral $\left(X^{2}{ }_{(2)}=56.00\right.$, $p<.001)$, el estado civil $\left(X^{2}{ }_{(4)}=32.31, p<.001\right)$, el consumo en el último año $\left(X^{2}{ }_{(1)}=21.10, p<.001\right)$ y el estatus de fumador $\left(X^{2}{ }_{(3)}=29.40, p<.001\right)$.

\section{Características de los fumadores regulares y ocasio- nales}

Se encontraron 86 personas que declararon fumar por lo menos un cigarro al día; su promedio de edad es de 32.1 años (DE=7.9); más de la mitad es del sexo masculino (60.5\%); la mayoría tiene una escolaridad mínima de bachillerato $(M=12.9)$ y una antigüedad en el hospital de 7.1 años ( $D E=5.9)$; la mayoría está casada o vive en unión libre (59.0\%).

Los 154 participantes que declararon ser fumadores ocasionales presentaron las siguientes características: una media de edad de 29.6 años (DE= 5.8) y un prome-

Tabla 2

Características de los fumadores del hospital y puntajes de dependencia

\begin{tabular}{|c|c|c|c|c|c|}
\hline & $\begin{array}{l}\text { Regulares } \\
\mathrm{n}=86\end{array}$ & & $\begin{array}{l}\text { Ocasionales } \\
n=154\end{array}$ & $\begin{array}{l}\text { Total fumadores } \\
n=240\end{array}$ & $\begin{array}{l}\text { Dif. } \\
\text { estadística }\end{array}$ \\
\hline & $M(D E)$ & & $M(\mathrm{DE})$ & $M(\mathrm{DE})$ & \\
\hline Edad: & $32.1(7.9)$ & & $29.6(5.8)$ & $30.5(6.8)$ & $t_{(232)}=2.72^{*}$ \\
\hline Antigüedad (años) & $7.1(5.9)$ & & $5.2(4.6)$ & $5.9(5.2)$ & $t_{(226)}=2.65^{\star}$ \\
\hline Años de estudio & $12.9(4.5)$ & & $12.7(3.4)$ & $12.8(3.9)$ & $t_{(211)}=.50$ \\
\hline \multirow[t]{2}{*}{ Edad de primer consumo } & $17.95(4.5)$ & & $17.3(2.8)$ & $17.3(3.6)$ & $t_{(226)}=1.20$ \\
\hline & $\mathrm{n}(\%)$ & & $\mathrm{n}(\%)$ & $n(\%)$ & \\
\hline \multicolumn{6}{|l|}{ Sexo } \\
\hline Mujer & $34(39.5)$ & & $73(48.7)$ & $107(45.3)$ & $X^{2}(1)=1.84$ \\
\hline Hombre & $52(60.4)$ & & $77(51.3)$ & $129(54.7)$ & \\
\hline \multicolumn{6}{|l|}{ Escolaridad } \\
\hline Primaria & $2(2.6)$ & & $2(1.4)$ & $4(1.9)$ & \\
\hline Secundaria & $19(24.3)$ & & $29(21.0)$ & $48(22.2)$ & \\
\hline Bachillerato & $34(43.6)$ & & $76(55.1)$ & $110(50.9)$ & $X^{2}(4)=2.99$ \\
\hline Licenciatura & $13(16.7)$ & & $19(13.8)$ & $32(14.8)$ & \\
\hline Posgrado & $10(12.8)$ & & $12(8.7)$ & $22(10.2)$ & \\
\hline \multicolumn{6}{|l|}{ Tipo de actividad laboral } \\
\hline Médicos & $9(10.5)$ & & $9(5.8)$ & $18(7.5)$ & \\
\hline Salud no médicos & $29(33.7)$ & & $49(31.8)$ & $78(32.5)$ & $X^{2}(2)=2.02$ \\
\hline Servicios y administrativos & $48(55.8)$ & & $96(62.3)$ & $144(60.0)$ & \\
\hline \multicolumn{6}{|l|}{ Estado civil } \\
\hline Soltero & $30(36.1)$ & & $57(39.0)$ & $87(38.0)$ & \\
\hline Casado/unión libre & $49(59.0)$ & & $87(59.6)$ & $136(59.4)$ & $X^{2}(2)=2.52$ \\
\hline \multirow[t]{2}{*}{ Separado/divorciado/viudo } & $4(4.8)$ & & $2(1.4)$ & $6(2.6)$ & \\
\hline & $M(\mathrm{DE})$ & IC 95\% & & & \\
\hline \multicolumn{6}{|l|}{ Puntajes de dependencia ${ }^{a}$} \\
\hline FTND $^{\mathrm{b}}$ & $.98(1.9)$ & $.53-1.53$ & & & \\
\hline $\mathrm{CDS}^{\mathrm{C}}-12$ & $25.73(5.5)$ & 23.91-27.58 & & & \\
\hline $\operatorname{CDS}^{\mathrm{C}}-5$ & $9.73(2.8)$ & $8.90-10.61$ & & & \\
\hline \multicolumn{6}{|c|}{${ }^{*} p<.05$} \\
\hline \multicolumn{6}{|c|}{ a Sólo se aplicó al grupo de consumo regular } \\
\hline${ }^{b}$ Fagerström Test of Nicotine Depen & dence & & & & \\
\hline \multicolumn{6}{|l|}{${ }^{\mathrm{c}}$ Cigarette Dependence Scale } \\
\hline
\end{tabular}


dio de 12.7 años de estudio; la mayoría (55.1\%) tiene estudios de bachillerato; $59.6 \%$ de ellos son casados o vive con su pareja (Tabla 2). Se encontraron diferencias estadísticamente significativas en edad $\left(t_{(232)}=2.72\right.$, $p=.007)$ y años de antigüedad $\left(t_{(226)}=2.65, p=.009\right)$ entre fumadores regulares y ocasionales (Tabla 2 ).

La media de calificación en las pruebas de dependencia de nicotina -las cuales sólo se aplicaron a fumadores regulares- fue de .98 ( $D E=1.98)$ en el FTND, 25.73 ( $D E=5.58)$ en la CDS-versión de 12 ítems y 9.73 ( $D E=2.8)$ en la Escala de CDS-versión de cinco ítems. 91.2\% de los fumadores presentó un nivel de dependencia muy bajo.

\section{Características de los médicos, personal de salud no médico y otro personal}

Los médicos que participaron en el estudio tienen un promedio de edad de 38.3 años (DE=7.4) y una media de 22.0 años ( $\mathrm{DE}=3$ ) de estudio; $58.9 \%$ es del género masculino; $88.4 \%$ tiene estudios de posgrado; $64.5 \%$ está casado; y poco más de la mitad es no fumadora (56.7\%).

El personal de salud reportó un promedio de edad de 32.4 años ( $\mathrm{DE}=7.3)$ y 14.0 años $(\mathrm{DE}=2.9)$ de estudio; $59.6 \%$ es de sexo femenino; $37 \%$ tiene escolaridad de bachillerato; $51.6 \%$ está casado; $55.2 \%$ es no fumador.

El personal administrativo y de servicios generales tiene una media de 29.7 años edad ( $D E=6.2$ ) y 11.5 años $(\mathrm{DE}=2.3$ ) de estudio; $55.3 \%$ es de sexo femenino; $55 \%$ estudió el bachillerato; $47.4 \%$ es no fumador. El resto de los datos demográficos y de consumo se pueden observar en la Tabla 3.

Se encontraron diferencias estadísticamente significativas entre los tres grupos en las variables de edad $\left(F_{(2,947)}=67.01, p<.001\right)$, antigüedad $\left(F_{(2,928)}=22.12\right.$, $p<.001)$, años de estudio $\left(F_{(2,863)}=582.98, p<.001\right)$,

Tabla 3

Comparación entre el personal de las diferentes áreas del hospital

\begin{tabular}{|c|c|c|c|c|c|}
\hline & Médicos & $\begin{array}{l}\text { Salud } \\
\text { no médicos }\end{array}$ & $\begin{array}{l}\text { Administrativo y } \\
\text { servicios }\end{array}$ & Total & $\begin{array}{l}\text { Dif. } \\
\text { estadística }\end{array}$ \\
\hline & $n=124$ & $n=392$ & $\mathrm{n}=488$ & $n=1004$ & \\
\hline & $M(\mathrm{DE})$ & $M(\mathrm{DE})$ & $M(\mathrm{DE})$ & $M(\mathrm{DE})$ & \\
\hline Edad: & $38.3(7.4)$ & $32.4(7.3)$ & $29.7(6.2)$ & $32.1(7.4)$ & $F_{(2,947)}=67.01^{*}$ \\
\hline Antigüedad (años) & $9.6(7.2)$ & $8.2(6.7)$ & $5.7(4.9)$ & $7.3(6.3)$ & $F_{(2,928)}=22 \cdot 12^{*}$ \\
\hline \multirow[t]{2}{*}{ Años de estudio } & $22.0(3.0)$ & $14.0(2.9)$ & $11.5(2.3)$ & $13.8(4.3)$ & $F_{(2,863)}=582.98^{\star}$ \\
\hline & $\mathrm{n}(\%)$ & $\mathrm{n}(\%)$ & $\mathrm{n}(\%)$ & $\mathrm{n}(\%)$ & \\
\hline \multicolumn{6}{|l|}{ Sexo } \\
\hline Mujer & $51(41.1)$ & $250(59.6)$ & $261(55.3)$ & $562(57.3)$ & $X^{2}{ }_{(2)}=25.10^{*}$ \\
\hline Hombre & $73(58.9)$ & $131(40.4)$ & $214(44.7)$ & $418(42.7)$ & \\
\hline \multicolumn{6}{|l|}{ Escolaridad } \\
\hline Primaria & 0 & 0 & $4(.9)$ & $4(.4)$ & \\
\hline Secundaria & 0 & $17(4.7)$ & $128(30.3)$ & $145(16.1)$ & \\
\hline Bachillerato & 0 & $180(50.3)$ & $226(53.4)$ & $406(45.0)$ & $X^{2}(8)=608.30^{*}$ \\
\hline Licenciatura & $14(11.6)$ & $117(32.7)$ & $54(12.8)$ & $185(20.5)$ & \\
\hline Posgrado & $107(88.4)$ & $44(12.3)$ & $11(2.6)$ & $162(18.0)$ & \\
\hline \multicolumn{6}{|l|}{ Estado civil } \\
\hline Soltero & $37(30.6)$ & $163(44.7)$ & $203(45.0)$ & $403(43.0)$ & \\
\hline Casado/unión libre & $80(66.1)$ & $199(54.5)$ & $239(53.0)$ & $518(55.3)$ & $X^{2}(4)=11.74^{*}$ \\
\hline Separado/divorciado/viudo & $4(3.3)$ & $3(.8)$ & $9(2.0)$ & $16(1.7)$ & \\
\hline \multicolumn{6}{|l|}{ Tipo de consumo } \\
\hline Fumador regular & $9(7.5)$ & $29(7.9)$ & $48(10.5)$ & $86(9.1)$ & \\
\hline Fumador ocasiona & $9(7.5)$ & $49(13.4)$ & $96(21.0)$ & $154(16.2)$ & $X^{2}(6)=20.83^{*}$ \\
\hline Exfumador & $34(28.3)$ & $88(24.0)$ & $96(21.0)$ & $218(23.0)$ & \\
\hline No fumador & $68(56.7)$ & $201(54.8)$ & $218(47.6)$ & $487(51.5)$ & \\
\hline
\end{tabular}

* $p<.05$ 
sexo $\left(X^{2}{ }_{(2)}=25.10, p<.001\right)$, escolaridad $\left(X^{2}{ }_{(8)}=608.30\right.$, $p<.001)$, estado civil $\left(X^{2}{ }_{(4)}=11.74, p=.019\right)$ y tipo de consumo $\left(X^{2}(6)=20.83, p=.002\right)$.

\section{Factores de riesgo relacionados con el consumo de tabaco}

Los participantes que tienen una mayor probabilidad de ser consumidores regulares, se encuentran en el grupo de edad de 20 a 31 años (OR=2.62, IC del 95\% [1.773.88], $p<.001$ ); son hombres (OR=2.91, IC del 95\% [1.824.68], $p<.001)$; relacionado con la edad de inicio del consumo (OR=1.09, IC del 95\% [.71-1.68], p<.001); están separados, divorciados o viudos (OR $=4.51$, IC del $95 \%$ [1.18-17.18], $p=.027)$; y su máximo nivel de estudios es de secundaria (OR=3.51, IC del 95\% [1.66-7.40], $p=.001)$ o bachillerato (OR=3.11, IC del 95\% [1.61-6.02], $p=.001)$.
También se identificó que trabajar en servicios y administración (OR=2.04, IC del 95\% [1.41-2.96], p<.001), tener escolaridad de licenciatura (OR=1.44, IC del 95\% [.66-3.13], $p=.032$ ) y el tiempo de servicio (OR=.913, IC del 95\% [.88-.94], $p<.001$ ) aumenta la probabilidad de ser consumidor ocasional (Tabla 4).

\section{DISCUSIÓN Y CONCLUSIONES}

El presente estudio tuvo como primer objetivo describir las características de consumo de la población de trabajadores de un hospital de tercer nivel; se encontró una prevalencia de $25.4 \%$ de consumidores de tabaco, similar a la reportada en la Ciudad de México (30.8\%), pero mayor en comparación con la población en general (21.7\%, Encuesta Nacional de Adicciones [ENA], 2011).

El porcentaje de fumadores regulares resultó ser similar

Tabla 4

Regresión multinomial en el consumo de tabaco y variables de exposición

\begin{tabular}{|c|c|c|c|c|c|c|c|c|}
\hline & Regular $^{\mathrm{a}}$ & Ocasional $^{a}$ & Exfumador $^{a}$ & $x^{2}$ & $g l$ & $p$ & OR & IC 95\% \\
\hline \multicolumn{9}{|l|}{ Grupo de edad } \\
\hline $44-56$ & b & $\mathrm{b}$ & $\mathrm{b}$ & b & b & $\mathrm{b}$ & $b$ & b \\
\hline $20-31$ & 1.20 & .965 & .475 & 23.29 & 1 & 000 & 2.62 & $1.77-3.88$ \\
\hline $32-43$ & -.215 & -.745 & -.496 & 8.16 & 1 & 004 & .60 & $.434-.856$ \\
\hline \multicolumn{9}{|l|}{ Sexo } \\
\hline Mujer & b & b & $\mathrm{b}$ & b & b & b & $b$ & b \\
\hline Hombre & 1.07 & .70 & .432 & 19.80 & 1 & 000 & 2.91 & $1.82-4.68$ \\
\hline \multicolumn{9}{|l|}{ Tipo de actividad laboral } \\
\hline Médicos & $\mathrm{b}$ & b & $\mathrm{b}$ & b & b & $\mathrm{b}$ & b & b \\
\hline Salud no médicos & -.323 & -.41 & -.04 & 4.36 & 1 & .037 & .66 & $.452-.975$ \\
\hline Servicios y administrativos & .44 & .714 & -03 & 14.18 & 1 & 000 & 2.04 & $1.41-2.96$ \\
\hline Edad de inicio de consumo & .089 & .042 & -.010 & 13.18 & 1 & 000 & 1.09 & $.710-1.68$ \\
\hline \multicolumn{9}{|l|}{ Estado civil } \\
\hline Casado/unión libre & $\mathrm{b}$ & b & $\mathrm{b}$ & $\mathrm{b}$ & b & $b$ & b & b \\
\hline Soltero & -.207 & .133 & -.064 & 5.56 & 1 & .024 & 1.131 & $.768-1.66$ \\
\hline Separado/divorciado/viudo & 1.50 & .214 & .777 & 4.89 & 1 & .027 & 4.51 & $1.18-17.18$ \\
\hline \multicolumn{9}{|l|}{ Escolaridad } \\
\hline Primaria & $\mathrm{b}$ & b & b & $\mathrm{b}$ & $\mathrm{b}$ & $\mathrm{b}$ & b & b \\
\hline Secundaria & 1.015 & 1.25 & -.259 & 10.85 & 1 & 001 & 3.51 & $1.66-7.40$ \\
\hline Bachillerato & .514 & 1.13 & -.060 & 11.41 & 1 & 001 & 3.11 & $1.61-6.02$ \\
\hline Licenciatura & .167 & .364 & -.139 & 7.38 & 1 & .032 & 1.44 & $.662-3.131$ \\
\hline Posgrado & -.576 & -.1 .01 & .116 & 9.69 & 1 & .002 & .36 & $.193-.688$ \\
\hline Tiempo en el hospital & -.026 & -.091 & -.026 & 22.52 & 1 & 000 & .91 & $.880-.948$ \\
\hline
\end{tabular}

Nota. OR= Razón de Momios; IC= Intervalo de Confianza; gl= Grados de Libertad

a Se utilizó el grupo de no fumadores como grupo de comparación

b Parámetro redundante y comparador 
al de la población general (9.1\% vs 8.9\%, según la ENA, 2011); $44.7 \%$ pertenece al área de salud.

La mayoría de los fumadores son consumidores ocasionales (16.2\%); si se considera que $27.2 \%$ de los fumadores ocasionales consume entre dos a tres veces por semana, la probabilidad de que muchos de estos progresen a un consumo regular es considerable (Nelson, Van Ryzin, \& Dishion, 2015; Orpinas, Lacy, Nahapetyan, Dube, \& Song, 2016), lo que aumenta la probabilidad de mortalidad por enfermedades relacionadas con el consumo de tabaco (Rosenberg et al., 2012).

El segundo objetivo fue comparar las distintas áreas de trabajo en el hospital, en función de variables relacionadas con el consumo de tabaco. Un hallazgo importante fue la baja prevalencia de consumo en el último año entre los médicos, lo que coincide con lo reportado por estudios previos entre médicos y enfermeras (Salmerón-Castro et al., 2002; Tong et al., 2010), trabajadores de salud y de otras áreas (Lewis et al., 2011). Una posible explicación es que en los últimos años se ha presentado una mayor exposición a información relacionada con el riesgo a la salud que implica el tabaquismo, además, se han implementado diversas políticas vinculadas con el marco legislativo (Martínez et al., 2016; OMS, 2003).

Para responder al tercer objetivo -identificar los factores de riesgo relacionados con el consumo de tabaco-, los resultados de este estudio apoyan la evidencia previa que sugiere que los factores de riesgo asociados con el consumo regular de tabaco se relacionan con el sexo masculino (proporción de 2:1 con las mujeres) (Abdulateef et al., 2016). Los hombres se inician en el consumo a una menor edad que las mujeres (Villatoro-Velázquez et al., 2016), lo que propicia el mantenimiento del hábito de fumar en años posteriores (Khubaib, Shahid, Lodhi, Malik, \& Jan, 2016; Veeranki et al., 2016), aumenta la probabilidad de presentar problemas de consumo (Sabra, 2007; Stone et al., 2012) y genera un bajo éxito de los intentos de dejar de fumar a edades más tempranas (Jarvis, Cohen, Delnevo, \& Giovino, 2013).

Otro hallazgo importante es el relacionado al nivel de estudios, asociado de manera significativa con el consumo de tabaco; una probable explicación es que los años de estudio influyen en el uso de esta sustancia (Carter et al., 2015); además, se observó que en el hospital, el consumo aumenta si el área del profesional es no médica (Velasco-Contreras, 2013) y también si se es soltero o separado (Cho et al., 2008).

Debido al diseño del estudio, resultó imposible conocer si los fumadores ocasionales son consumidores de inicio tardío, es decir, si comenzaron a fumar a una edad mayor y continúan fumando, o si se iniciaron por variables relacionadas con el desarrollo (estilo de crianza, accesibilidad, ambiente), que marcan un inicio tardío y propician una pronta abstinencia natural (Brook, Balka, Ning, \& Brook, 2007); esto podría ser estudiado a través de un estudio de cohorte (Holford et al., 2014), en el que se evalúe el uso de tabaco en varias ocasiones en el tiempo y se indaguen más variables psicosociales e interpersonales que puedan establecer factores de riesgo y protección en la persona; asimismo, una investigación de este tipo ayudaría a determinar los años de vida perdidos o el aumento en la esperanza de vida.

Entre las principales limitaciones del estudio se enumeran las siguientes: 1) se desarrolló únicamente en un centro: la utilización de varios centros permitiría obtener una muestra más amplia y resultados más generalizables; 2) el no contar con otro tipo de marcadores no permite constatar si las respuestas se deben a la deseabilidad social o a otro tipo de sesgo derivado del autorreporte; 3) no fue posible establecer un modelo estadístico que diera cuenta de los factores de riesgo en el uso de tabaco; 4) no se exploraron factores sociales asociados (por ejemplo, consumo del conyugue o familiares, expectativas de resultados, riesgo percibido, entre otros), lo cual limita en gran medida la información obtenida; 5) no se evaluaron las actitudes, los conocimientos y las prácticas del personal médico y de salud en torno al tabaquismo, lo que impide entender a mayor profundidad las implicaciones del uso del tabaco en las prácticas antitabaco de estos profesionales; y 6) los recursos limitados, en particular la falta de personal específicamente capacitado para la aplicación del instrumento, influenció mucho en la pérdida de datos, así como en el riesgo elevado de sesgos.

No obstante a estas limitaciones, la principal aportación del estudio es contribuir con información actual del consumo de tabaco y con variables relacionadas con su uso por parte del personal del hospital, lo cual podría orientar el establecimiento de programas de prevención para evitar que los fumadores ocasionales (gran parte de ellos entre los 20 y 31 años) progresen a patrones de uso más intensivos; se espera, además, contribuir al desarrollo de políticas específicas para impactar a este grupo de edad, el cual podría estar mediado por el nivel de estudios, sin embargo, los datos obtenidos no son conclusivos y es necesario llevar a cabo más investigación (con mayor control de sesgos y con diseños) en torno al impacto estimado de dichas estrategias en la población mexicana. Por último, es de suma importancia conocer la forma en que se distribuye el consumo entre los trabajadores del hospital, para la implementación de estrategias que permitan optimizar la utilización de recursos en la prevención y el tratamiento del tabaquismo, a fin de evitar que el personal de las instituciones de salud se conviertan en un modelo negativo para los pacientes. 


\section{FUENTES DE FINANCIAMIENTO}

Durante la conducción del estudio LVG se obtuvo financiamiento por parte del Consejo Nacional de Ciencia y Tecnología (CONACYT), a través de la beca núm. 303501.

\section{CONFLICTOS DE INTERÉS}

Los autores de este artículo declaramos que no existe ningún conflicto de interés en la realización de este manuscrito.

\section{REFERENCIAS}

Abdulateef, D. S., Ali, A. J., Abdulateef, D. S., \& Mohesh, M. I. G. (2016). Smoking knowledge, attitude, and practices among health care professionals from Sulaymaniyah City/lraq. Tobacco Use Insights, 9, 1-6. doi: 10.4137/TUI.S38171

Al-Hosani, S., Al-Ali, M., Al-Marashda, K., Al-Shamsi, N., Al-Ansari, T., Al-Behandy, A., ... Elhassan, A. (2014). Smoking prevalence, attitudes and behaviors primary healthcare providers and its impact on their smoking cessation counseling practices. Ibnosina Journal of Medicine and Biomedical Sciences, 7(2), 47-55. Recuperado de http://journals.sfu.ca/ijmbs/index.php/ijmbs/article/ view/528

Arenas, L., Hernández, I., Jasso, R., Martínez, P., \& Menjivar, A. (2004). Prevalencia de tabaquismo en médicos y enfermeras en los estados de Morelos y Guanajuato. Revista del Instituto Nacional de Enfermedades Respiratorias, 17(4), 261-265.

Brook, J. S., Balka, E. B., Ning, Y., \& Brook, D. W. (2007). Trajectories of cigarette smoking among African Americans and Puerto Ricans from adolescence to young adulthood: Associations with dependence on alcohol and illegal drugs. American Journal on Addictions, 16(3), 195-201. doi: 10.1080/10550490701375244

Carter, B. D., Abnet, C. C., Feskanich, D., Freedman, N. D., Hartge, P., Lewis, C. E., ... Jacobs, E. J. (2015). Smoking and mortality. Beyond established causes. New England Journal of Medicine, 372(7), 631-640. doi: 10.1056/NEJMsa1407211

Chapman, S. (1995). Doctors who smoke. BMJ (Clinical Research Ed.), 311(6998), 142-143. Recuperado de http://www.ncbi.nlm. nih.gov/pubmed/7613415

Cho, H. J., Khang, Y. H., Jun, H. J., \& Kawachi, I. (2008). Marital status and smoking in Korea: The influence of gender and age. Social Science \& Medicine, 66(3), 609-619. doi: 10.1016/j.socscimed.2007.10.005

Duaso, M. J., McDermott, M. S., Mujika, A., Purssell, E., \& While, A. (2014). Do doctors' smoking habits influence their smoking cessation practices? A systematic review and meta-analysis. Addiction, 109(11), 1811-1823. doi: 10.1111/add.12680

Etter, J. F., Le Houezec, J., \& Perneger, T. V. (2003). A self-administered questionnaire to measure dependence on cigarettes: the cigarette dependence scale. Neuropsychopharmacology, 28(2), 359-370. doi: 10.1038/sj.npp. 1300030
Guerrero-López, C. M., Reynales-Shigematsu, L. M., Jiménez-Ruiz, J. A., Karam-Araujo, R., Maldonado-Cruz, C. A., \& Camacho-Solís, R. (2012). Costos por ausentismo laboral atribuibles al consumo de tabaco en el Instituto Mexicano del Seguro Social y en México, 2006-2009. Salud Pública de México, 54(3), 233-241.

Heatherton, T. F., Kozlowski, L. T., Frecker, R. C., \& Fagerstrom, K. O. (1991). The Fagerstrom Test for Nicotine Dependence: a revision of the Fagerstrom Tolerance Questionnaire. British Journal of Addiction, 86, 1119-1127.

Holford, T. R., Meza, R., Warner, K. E., Meernik, C., Jeon, J., Moolgavkar, S. H., ... Levy, D. T. L. (2014). Tobacco control and the reduction in smoking-related premature deaths in the United States, 1964-2012. Journal of the American Medical Association, 311(2), 164. doi: 10.1001/jama.2013.285112

Jarvis, M. J., Cohen, J. E., Delnevo, C. D., \& Giovino, G. A. (2013). Dispelling myths about gender differences in smoking cessation: Population data from the USA, Canada and Britain. Tobacco Control, 22(5), 356-360. doi: 10.1136/tobaccocontrol-2011050279

Khubaib, M. U., Shahid, Z. Y., Lodhi, S. K., Malik, H., \& Jan, M. M. (2016). Prevalence and associated factors of smoking among final year medical students: A multicentric survey from Pakistan. Cureus, 8(7), e701. doi: 10.7759/cureus.701

Lewis, K. E., Shin, D., \& Davies, G. (2011). Smoking habits and attitudes toward tobacco bans among United Kingdom hospital staff and students. The International Journal of Tuberculosis and Lung Disease: The Official Journal of the International Union against Tuberculosis and Lung Disease, 15(8), 1122-1126. doi: 10.5588/ijtld. 10.0783

Maeckelberghe, E. L. M. (2013). Doctors and medical students as non-smoking role models? Using the right arguments. European Journal of Public Health, 23(2), 190-192. doi: 10.1093/eurpub/ ckt003

Martínez, C., Martínez-Sánchez, J. M., Antón, L., Riccobene, A., Fu, M., Quirós, N., ... Fernández, E. (2016). Prevalencia de consumo de tabaco en trabajadores hospitalarios: Metaanálisis en 45 hospitales catalanes. Gaceta Sanitaria, 30(1), 55-58. doi: 10.1016/j.gaceta.2015.08.006

Murray, C. J. L., \& Lopez, A. D. (1997). Global mortality, disability, and the contribution of risk factors: Global burden of disease study. Lancet, 349(9063), 1436-1442. doi: 10.1016/S01406736(96)07495-8

Nelson, S. E., Van Ryzin, M. J., \& Dishion, T. J. (2015). Alcohol, marijuana, and tobacco use trajectories from age 12 to 24 years: Demographic correlates and young adult substance use problems. Development and Psychopathology, 27(1), 253-277. doi: 10.1017/S0954579414000650

Organización Mundial de la Salud. (2015). WHO report on the global tobacco epidemic, 2015. Ginebra: Autor

Organizacion Mundial de la Salud. (2003). Convenio marco de la OMS para el Control del Tabaco. Ginebra: Autor. Recuperado de www.who.int/fctc 
Orozco-González, C. N., Cortés-Sanabria, L., Viera-Franco, J. J., Ramírez-Márquez, J. J., \& Cueto-Manzano, A. M. (2016). Prevalencia de factores de riesgo cardiovascular en trabajadores de la salud. Revista Médica del Instituto Mexicano del Seguro Social, 54(5), 594-601.

Orpinas, P., Lacy, B., Nahapetyan, L., Dube, S. R., \& Song, X. (2016). Cigarette smoking trajectories from sixth to twelfth grade: Associated substance use and high school dropout. Nicotine and Tobacco Research, 18(2), 156-162. doi: 10.1093/ntr/ntv040

Pipe, A., Sorensen, M., \& Reid, R. (2009). Physician smoking status, attitudes toward smoking, and cessation advice to patients: An international survey. Patient Education and Counseling, 74(1), 118-123. doi: 10.1016/j.pec.2008.07.042

Ravara, S. B., Castelo-Branco, M., Aguiar, P., \& Calheiros, J. M. (2014). Are physicians aware of their role in tobacco control? A conference-based survey in Portugal. BMC Public Health, 14, 979. doi: 10.1186/1471-2458-14-979

Reynales-Shigematsu, L. M., Guerrero-López, C. M., Lazcano-Ponce, E., Villatoro-Velázquez, J. A., Medina-Mora, M. E., Fleiz-Bautista, C., ... (2012). Encuesta Nacional de Adicciones 2011: Reporte de Tabaco. México, D.F., México: INPRF. Recuperado de www.inprf.gob.mx, www.conadic.gob.mx, www.cenadic.salud. gob.mx, www.insp.mx

Röhrig, B., du Prel, J. B., Wachtlin, D., \& Blettner, M. (2009). Types of study in medical research: Part 3 of a series on evaluation of scientific publications. Deutsches Ärzteblatt International, 106(15), 262-268. doi: 10.3238/arztebl.2009.0262

Rosenberg, M. A., Feuer, E. J., Yu, B., Sun, J., Henley, S. J., Shanks, T. G., .. Burns, D. M. (2012). Chapter 3: Cohort life tables by smoking status, removing lung cancer as a cause of death. Risk Analysis, 32(s1), S25-S38. doi: 10.1111/j.15396924.2011.01662.x

Sabra, A. A. (2007). Smoking attitudes, behaviours and risk perceptions among primary health care personnel in urban family medicine centers in Alexandria. The Journal of the Egyptian Public Health Association, 82(1-2), 43-64. Recuperado de http://www. ncbi.nlm.nih.gov/pubmed/18217324
Salmerón-Castro, J., Arillo-Santillán, E., Campuzano-Rincón, J. C., López-Antuñano, F. J., \& Lazcano-Ponce, E. C. (2002). Tabaquismo en profesionales de la salud del Instituto Mexicano del Seguro Social, Morelos. Salud Pública de México, 44, s67-s75. doi: 10.1590/S0036-36342002000700011

Stone, A. L., Becker, L. G., Huber, A. M., \& Catalano, R. F. (2012). Review of risk and protective factors of substance use and problem use in emerging adulthood. Addictive Behaviors, 37(7), 747-775. doi: 10.1016/j.addbeh.2012.02.014

Tong, E. K., Strouse, R., Hall, J., Kovac, M., \& Schroeder, S. A. (2010). National survey of U.S. health professionals' smoking prevalence, cessation practices, and beliefs. Nicotine \& Tobacco Research: Official Journal of the Society for Research on Nicotine and Tobacco, 12(7), 724-33. doi: 10.1093/ntr/ntq071

Veeranki, S. P., John, R. M., Ibrahim, A., Pillendla, D., Thrasher, J. F., Owusu, D., ... Mamudu, H. M. (2016). Age of smoking initiation among adolescents in Africa. International Journal of Public Health, 1-10. doi: 10.1007/s00038-016-0888-7

Velasco-Contreras, M. E. (2013). Perfil de salud de los trabajadores del Instituto Mexicano del Seguro Social. Revista Médica del Instituto Mexicano del Seguro Social, 51(1), 12-25.

Villatoro-Velázquez, J. A., Medina-Mora, M. E., Martín del Campo, R., Ito, D. A., Fregoso-Gamiño, M. N. B., Escobar, E. R., .. Cañas-Martínez, V. (2016). El consumo de drogas en estudiantes de México: tendencias y magnitud del problema. Salud Mental, 39(4), 193-203.

Waters, H., Sáenz-de Miera, B., Ross, H., \& Reynales-Shigematsu, L. (2010). La economía del tabaco y los impuestos al tabaco en México. París: Unión Internacional contra la Tuberculosis y Enfermedades Respiratorias 